\title{
Preface: Joint researches are benefiting the Chang'E-1 compre- hensive lunar scientific studies which probe ever deeper
}

Payloads of the first Chinese lunar mission Chang'E-1 obtained fruitful scientific data which cover a wide range of disciplines and fields. From 2007, many domestic research teams have been working hard on calibrating and validating these data cautiously and carefully. They have made out a lot of outstanding presentations and publications in various scientific meetings and journals. Recently, these Chinese lunar science pioneers found many more deep research results by means of collaborations between/among teams, by combining the data from the new lunar missions.

With the strong support from SCEINCE CHINA Physics, Mechanics \& Astronomy, a total of six papers have been collected in this special issue, all in both of Chinese and English, with the agreements from the authors. Among them, results from two papers were published by combining the data from the Lunar Orbiter, Clementine, Lunar Reconnaissance Orbiter (LRO), Kaguya/SELENE and Chang'E-1 missions. In the papers, Xiao et al. studied the origin of pit chains in the floor of lunar Copernican Craters together with an international team, and Chen et al. carried out a geologic investigation and mapping of the Sinus Iridum quadrangle with another international team. There are two papers here that used the data from the payload of Chang'E-1 interference imaging spectrometer (IIM), one which discusses the Global Absorption Center Map of the Mafic Minerals on the Moon by Wu et al., and the other discusses the lunar titanium content, after carefully processing the IIM data. These two teams have not only contributed to the new lunar discoveries, but also contributed a lot to validating and calibrating the IIM data. To map the water ice content on the lunar surface is by no means easy work with poor data, however Meng and his international team tried their best to calibrate the data and finally found some meaningful clues in the Cabeus Crater by using the data from the Microwave Radiometer onboard Chang'E-1. Additionally, in the Japanese lunar explorer SELENE (KAGUYA), 3 out of the 14 onboard instruments took selenodetic measurements. In addition, the Chinese VLBI network took part in the VLBI lunar gravity mission of SELENE. The mission PI, senior visiting professor of the Chinese Academy of Sciences, Prof. Nobuyuki Kawano, went over this scientific story in a review paper of the results obtained by geodetic instruments of SELENE (KAGUYA), together with his three Chinese colleagues (Co-I).

The relevant papers and scientific results from the Chang'E-1 lunar orbiter that appear in this special issue will not only benefit the lunar science study and future lunar exploration, but also show an outstanding example of the how international collaboration will benefit the Chinese lunar and deep space exploration(s).

PING JinSong

Special Topic Editor

Shanghai Astronomical Observatory, Chinese Academic of Sciences

November, 2010 